\title{
Arbor
}

\section{Presentación de la obra de H. Poincaré (1854-1912)}

\section{Manuel López Pellicer}

Arbor CLXXVIII, 704 (Agosto 2004), 621-624 pp.

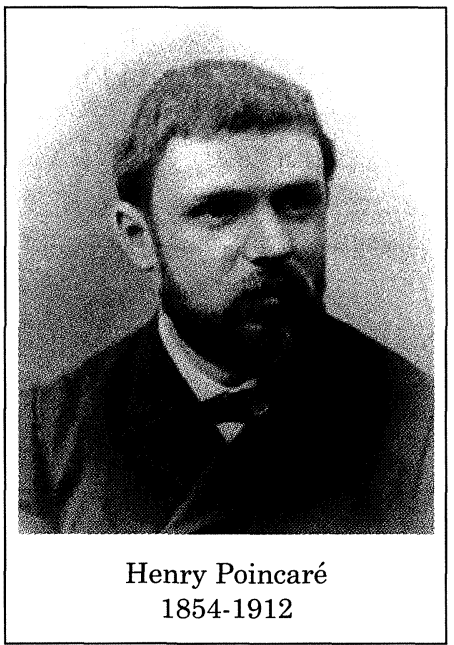

La Sección de Exactas de la Real Academia de Ciencias, dentro de las actividades del curso 2003-2004, ha conmemorado el sesquicentenario del nacimiento del célebre matemático francés Henri Poincaré con un ciclo de conferencias dado el 27 de mayo de 2004 en su sede, que ha contado con la intervención de los profesores de la Universidad de Valladolid José Manuel Aroca Hernández-Ros, Felipe Cano Torres y Javier de Lorenzo Martínez, del profesor de la Universidad Politécnica de Cataluña Amadeu Delshams i Valdés y de la profesora de la Universidad de Zaragoza María Teresa Lozano Imízcoz. La Sección agradece a estos profesores su interés, dedicación y excelente trabajo, y, en particular, al profesor José Manuel Aroca, académico de esta Real Academia de Ciencias, su sugerencia de conmemorar esta efeméride. También debemos, y deseamos, hacer constar la gran ayuda del académico José María Montesinos en el diseño de este ciclo de conferencias en honor de Henri Poincaré.

Poincaré nació el 29 de abril de 1854 en Nancy y falleció el 17 de julio de 1912 en París. Su padre, Léon Poincaré, era profesor en la Facultad de Medicina de Nancy y pertenecía a una familia distinguida de Francia. Raymond Poincaré, varias veces Primer Ministro y Presidente de la República Francesa durante la Primera Guerra Mundial, era primo hermano de Henry Poincaré, quien, ambidiestro y con pobre coordinación muscular durante su niñez, recibió la instrucción especial de su culta madre y sobresalió en la composición escrita en la escuela primaria. 
En 1862 comenzó sus estudios en el Lycée en Nancy (hoy llamado Lycée Henri Poincaré en su honor) donde destacó en todas las materias, salvo en Educación Física y manualidades. Su profesor de Matemáticas le describió como un «monstruo de las matemáticas» y ganó los primeros premios de los Concursos Generales, competiciones donde participaban los mejores alumnos de todos los Lycées de Francia.

En 1875 se graduó en la Escuela Politécnica, donde demostró estar muy por delante de sus compañeros en Matemáticas, pero no en las asignaturas que exigían coordinación muscular. Poincaré tenía una memoria excelente, que enlazaba racionalmente todo lo que oía, y con la que suplía su deficiente visión en las conferencias que asistía. Se inscribió en l'École Polytechnique en 1873, y se graduó en 1875. Después continuó sus estudios en la Escuela de Minas y se doctoró en París en 1879, siendo Charles Hermite el director de su Tesis sobre Ecuaciones Diferenciales. Se le objetó confusión en la exposición, pero se le dio la máxima calificación por la dificultad de tema y el talento demostrados.

Tras enseñar dos años en la Universidad de Caen pasó, gracias al apoyo de Hermite, a París, a la Sorbona y a la Escuela Politécnica, para explicar Física Matemática y Probabilidad. En concreto se le encargó la óptica, la electricidad, el equilibrio de las masas flúidas, las matemáticas de la electricidad, astronomía, la termodinámica, la luz, y la probabilidad. Fue catedrático en la Sorbona y en la Escuela Politécnica de París hasta su muerte a los 58 años.

$\mathrm{Su}$ producción matemática fue abrumadora. Investigaba con gran regularidad cuatro horas diarias, entre las 10 y las 12 y luego, por la tarde, entre las 17 y 19 horas, evitando investigar más tarde para poder descansar bien. A última hora de la tarde y por la noche sólo leía artículos de investigación o libros. Así es como obtuvo importantes contribuciones en muchas ramas de la Matemática, de la Mecánica Celeste, de la Mecánica de Fluidos, de la Teoría especial de la Relatividad y de la Filosofía de la Ciencia.

Antes de los treinta años había desarrollado el concepto de función automorfa y había vuelto sobre su tesis doctoral con el objetivo de integrar todas las ecuaciones diferenciales lineales con coeficientes algebraicos. Sus cinco memorias sobre funciones y grupos fuchsianos, publicados en Acta matemática entre 1882 y 1884, junto con la veintena de notas en los Comptes Rendues constituyen una de las piezas más brillantes de las Matemáticas, descrita a continuación en el artículo Monodromía y ecuaciones fuchsianas en la obra de H. Poincaré, cuyo autor es el Profesor Aroca. 
Poincaré fue considerado en su época como el matemático más brillante del momento. Buena prueba de ello es que se le encargó la conferencia inaugural del Primer Congreso Internacional de Matemáticos, celebrado en Zurich en Agosto de $1897^{1}$. En ese congreso explicó las funciones que corresponden a un matemático, dando un programa, que se llama Programa Poincaré, relativo a como hacer Matemática no sólo para el momento, sino para el porvenir. La frase de Poincaré "en las cinco Memorias ..., no he hecho más que aflorar un tema muy amplio que proporcionará sin duda a los geómetras la ocasión de hacer numerosos e importantes descubrimientos" refleja el espíritu que trató de vivir con total honestidad toda su vida. El "Programa Poincaré" o Funciones del matemático da título a un artículo del profesor de Lorenzo, que lo pudimos oír en su conferencia en la Academia en la referida conmemoración y ahora leer en esta publicación.

En Matemática Aplicada, Poincaré estudió Capilaridad, Cosmología, Elasticidad, Mecánica Celeste, Óptica, Teoría Cuántica, Teorías de la luz y de las ondas electromagnéticas, Teoría de la Relatividad, Teoría del Potencial y Termodinámica. Se le considera co-descubridor con Albert Einstein y Hendrick Lorenz de la teoría especial de la relatividad. En este ciclo de conferencias, el profesor Amadeu Deshalms fue el autor de la conferencia "Poincaré, creador de los métodos todavía modernos en las ecuaciones diferenciales y en la mecánica celeste", donde se exponen algunas de sus contribuciones principales en Ecuaciones Diferenciales y en Mecánica Celeste, y se discute la importancia que tuvo la Memoria que presentó en 1889 sobre el problema de los tres cuerpos a un concurso, que lo ganó, convocado para conmemorar el sexagésimo aniversario del Rey Óscar II de Suecia y Noruega.

Cuando esta Memoria iba a ser publicada en Acta Mathematica, Phragmen, editor de Acta Matemática, descubrió un error, que significó el comienzo del intercambio de cincuenta cartas entre Poincaré y MittagLeffler, que se opuso a la publicación de la Memoria en tanto contuviese el error. En la última de esas cartas se discute la naturaleza del error, que se considera hoy como el origen del estudio del caos. Finalmente una versión revisada de la Memoria de Poincaré se publicó en 1890. Entre 1892 y 1899 Poincaré publicó tres tomos sobre Les Méthodes nouvelles de la mécanique céleste y en 1905 sus Leçons de mecanique céleste (1905).

Entre la últimas fechas citadas, Poincaré tuvo tiempo para la publicación de su obra Analysis situs en 1895, que le convirtió en el fundador de la Topología, uno de cuyos principales problemas es el reconocer cuando dos objetos son topológicamente equivalentes, lo que significa que uno 
se obtiene del otro por una deformación continua, sin singularidades, llamada isotopía. En las isotopías hay propiedades que permanencen y que se llaman invariantes. Poincaré ideó interesantes invariantes de naturaleza algebraica, que forman el núcleo de la Topología Algebraica, como el grupo fundamental o grupo de Poincaré, descrito en la última conferencia de esta publicación de la Profesora Lozano Imízcoz, en la que también expone la situación actual de la, tal vez, aún no resuelta Conjetura de Poincaré, a cuya solución también está esperando el premio prometido por el Instituto Clay. Consiste en probar que la propiedad que caracteriza topológicamente la esfera tridimensional es tener el grupo fundamental trivial.

Todo lo anterior constituye un material conmemorativo de gran interés y la Sección de Exactas, como no podía ser de otra manera, se congratula de su publicación en la Revista Arbor y agradece a la Comisión de Publicaciones de la Academia todo el apoyo prestado para la realización de esta publicación.

Finalmente, la Sección de Exactas reitera su reconocimiento y felicitación a todos los autores que han participado en este evento, y dado que la limitación temporal ha impedido tratar algunos temas físicos y matemáticos del trabajo de Poincaré, sería deseo de la Sección poder ampliar este trabajo antes del bicentenario de su nacimiento para lo que contamos, además de la colaboración de los profesores Aroca, Cano, de Lorenzo, Delshams y de la profesora Lozano, con las Secciones de Ciencias Exactas y de Ciencias Físicas y Químicas de la Real Academia de Ciencias de Madrid.

\section{Notas}

1 La conferencia inaugural del Segundo Congreso Internacional de Matemáticos del año 2000 en París fue dada por David Hilbert. Se ha dicho que Poincaré y Hilbert fueron los dos últimos matemáticos que dominaron toda la Matemática. Muchos opinan que a John von Neumann debe aplicársele la misma consideración. 